\title{
Quasi-ballistic-electron transport in random superlattices
}

\author{
Francisco Domínguez-Adame \\ Departamento de Física de Materiales, Facultad de Físicas, Universidad Complutense, E-28040 Madrid, Spain
}

Angel Sánchez and Enrique Diez

Escuela Politécnica Superior, Universidad Carlos III de Madrid, C./Butarque 15, E-28911 Leganés, Madrid, Spain

(Received 1 August 1994; revised manuscript received 20 September 1994)

\begin{abstract}
We theoretically study electron transport in disordered, quantum-well-based, semiconductor superlattices with structural short-range correlations. Our system consists of equal-width square barriers and quantum wells with two different thicknesses. The two kinds of quantum wells are randomly distributed along the growth direction. Structural correlations are introduced by adding the constraint that one of the wells always appears in pairs. We show that such correlated disordered superlattices exhibit a strong enhancement of their dc conductance as compared to usual random ones, giving rise to quasi-ballistic-electron transport. Interestingly, this phenomenon is also detected in superlattices with random fluctuations of the well thicknesses. Our predictions can be used to demonstrate experimentally that structural correlations inhibit the localization effects of disorder and, most important, that it should be clearly observed even in the presence of imperfections.
\end{abstract}

\section{INTRODUCTION}

It is by now well established on firm theoretical grounds that electron localization may be suppressed and bands of extended states appear in one-dimensional random systems with structural short-range correlations (see, e.g., Refs. 1 and 2 , and references therein). This unexpected phenomenon is not restricted to electronic systems but rather seems to be quite general, as it has also dramatic effects in classical harmonic chains, ${ }^{3,4}$ magnon propagation, ${ }^{5}$ or exciton dynamics. ${ }^{6}$ All these theoretical analyses contradict the earlier belief that all eigenstates are localized in one-dimensional disordered systems. ${ }^{7}$ Due to the lack of experimental confirmation, there is still some controversy as to the relevance of these results, their physical implications on transport properties, and the realizability of new devices based on those peculiar properties. Here we concern ourselves with semiconductor superlattices (SL's) in order to find experimentally measurable quantities and physically realizable systems that allow for a clearcut validation of the above-mentioned results. The reason for the choice of SL's for this purpose is twofold: First, they have been already used successfully to observe electron localization in intentionally uncorrelated disordered quantum-well (QW) based $\mathrm{GaAs} / \mathrm{Ga}_{1-x} \mathrm{Al}_{x} \mathrm{As}$ SL's. ${ }^{8}$ On the other hand, previous results of Diez, Sánchez, and Dominguez-Adame ${ }^{9}$ on simple, highly idealized models of SL's indicate that the effects of correlated disordered SL's should be clearly visible in such systems. These two reasons, and the fact that a number of studies have been performed to date on carrier dynamics in disordered SL's, ${ }^{10}$ make these systems the ideal candidates to propose experiments on localization or delocalization electronic properties. Accordingly, in this paper we deal with $\mathrm{GaAs} / \mathrm{Ga}_{1-x} \mathrm{Al}_{x}$ As SL's specifically designed for experiments on correlated disorder. In Sec. II we present our system and our analytical results on transport properties. Section III contains our numerical studies on electron transport through disordered SL's and the effect we will call quasi-ballistic-electron transport. After that, we address the question of whether the effect is really observable in a real system. To this end, we consider imperfect SL's by allowing the QW thicknesses to fluctuate around the ideal values and study how this unintentional randomness affects electron transport. Section IV ends the paper with a summary of our results and a brief discussion on how experimental measurements can be carried out.

\section{ANALYTICAL RESULTS}

\section{A. Electron dynamics in QWSL's}

The SL consists of two kinds of semiconductor layers (hereafter called $A$ and $B$ ) arranged alternatively along the growth direction $X$. Let $\Delta E_{c}$ be the conduction-band offset defined as $E_{c B}-E_{c A}$ and without loss of generality we will take $\Delta E_{c}>0$. In addition, we further consider that the thickness of layers $B$ is the same in the whole SL and denote it by $b$. Denoting by $x_{n}$ the spatial coordinate of the center of the $n$th barrier, the conduction-band profile is given as $V_{\mathrm{SL}}(x)=\Sigma_{n} V\left(x-x_{n}\right)$, where $V\left(x-x_{n}\right)$ takes the constant value $\Delta E_{c}$ for $\left|x-x_{n}\right|<b / 2$ and vanishes otherwise. We focus on electron states close to the band gap with $\mathbf{k}_{\|}=\mathbf{0}$ and use the one-band effective-mass framework to calculate the envelope functions

$$
\left[-\frac{\hbar^{2}}{2 m^{*}} \frac{d^{2}}{d x^{2}}+V_{\mathrm{SL}}(x)\right] F(x)=E F(x)
$$

where an explicit dependence of both $E$ and $F(x)$ on quantum numbers is understood and they will be omitted in the rest of the paper. We have taken a constant effective mass $m^{*}$ at the $\Gamma$ valley although this is not a serious limitation as our description can be easily generalized to include two different effective masses. Let us consider states below the barrier, which are of most interest to study quantum confinement effects. The corresponding envelope-function values at 
both sides of a barrier are related via a $2 \times 2$ transfer matrix $M(n)$ whose elements are $M_{11}(n)=M_{22}^{*}(n) \equiv \alpha_{n}$ and $M_{12}(n)=M_{21}^{*}(n) \equiv \beta_{n}$ where

$\alpha_{n}=\left[\cosh (\eta b)+i\left(\frac{\kappa^{2}-\eta^{2}}{2 \kappa \eta}\right) \sinh (\eta b)\right] \exp \left[i \kappa\left(\Delta x_{n}-b\right)\right]$

$\beta_{n}=-i\left(\frac{\kappa^{2}+\eta^{2}}{2 \kappa \eta}\right) \sinh (\eta b) \exp \left[-i \kappa\left(\Delta x_{n}-b\right)\right]$

with $\Delta x_{n} \equiv x_{n}-x_{n-1}, \kappa^{2}=2 m^{*} E / \hbar^{2}$, and $\eta^{2}=2 m^{*}(V-E) /$ $\hbar^{2}$. Letting $N$ be the total number of barriers, the transfer matrix of the SL is obtained as the product $T(N)=M(N) M N(N-1) \cdots M(1)$. The element $T_{11}(N)$ $\equiv A_{N}$ can be easily calculated recursively from the relationship ${ }^{2}$

$$
A_{n}=\left(\alpha_{n}+\alpha_{n-1}^{*} \frac{\beta_{n}}{\beta_{n-1}}\right) A_{n-1}-\left(\frac{\beta_{n}}{\beta_{n-1}}\right) A_{n-2},
$$

supplemented by the initial conditions $A_{0}=1, A_{1}=\alpha_{1}$. The knowledge of $A_{N}$ enables us to obtain the transmission coefficient $\tau$ at a given energy $E, \tau=\left|A_{N}\right|^{-2}$, and the singlechannel, dimensionless Landauer resistance, ${ }^{11} \rho=1 / \tau-1$ $=\left|A_{N}\right|^{2}-1$. Finally, the dimensionless Lyapunov coefficient is a non-negative parameter given by ${ }^{12} \gamma=-(1 / 2 N) \ln \tau$, being nothing but the inverse of the localization length in units of the SL period.

\section{B. Transmission through a single DQW}

We now consider a single dimer quantum well (DQW), with the $k$ th barrier in between, in an otherwise periodic SL. We denote the thickness of the QW in the periodic SL by $a$ whereas the thickness of each QW forming the DQW is denoted by $a^{\prime}$. The condition for an electron to move in the periodic SL is $|\operatorname{Tr}[M(1)]| \leqslant 2$ and the corresponding minibands are

$\left|\cos (\kappa a) \cosh (\eta b)-\left(\frac{\kappa^{2}-\eta^{2}}{2 \kappa \eta}\right) \sin (\kappa a) \sinh (\eta b)\right| \leqslant 1$.

For brevity we put $\alpha_{k}=\alpha_{k+1} \equiv \alpha^{\prime}$ and $\alpha_{n} \equiv \alpha(n \neq k, k+1)$. Considering Eq. (3) for $n=k, k+1, k+2$, eliminating $A_{k}$ and $A_{k+1}$, and setting $\operatorname{Re}\left(\alpha^{\prime}\right)=0$ we obtain after a little algebra

$$
-A_{k+2}=\left(\alpha+\alpha^{*}\right) A_{k-1}-A_{k-2} .
$$

Besides a constant phase factor of $\pi$ which has no effects on the magnitudes of interest, Eq. (5) reduces to Eq. (3) for a periodic SL in which sites $k$ and $k+1$ have been eliminated. This means that the reflection coefficient at the DQW vanishes and, consequently, there exists complete transparency at the resonant energy $E_{r}$ satisfying $\operatorname{Re}\left(\alpha^{\prime}\right)=0$, i.e.,

$$
\cos \left(\kappa_{r} a^{\prime}\right) \cosh \left(\eta_{r} b\right)-\left(\frac{\kappa_{r}^{2}-\eta_{r}^{2}}{2 \kappa_{r} \eta_{r}}\right) \sin \left(\kappa_{r} a^{\prime}\right) \sinh \left(\eta_{r} b\right)=0
$$

where the subscript $r$ refers to the resonant energy $E_{r}$. Importantly, choosing $a^{\prime}$ appropriately allows us to locate the

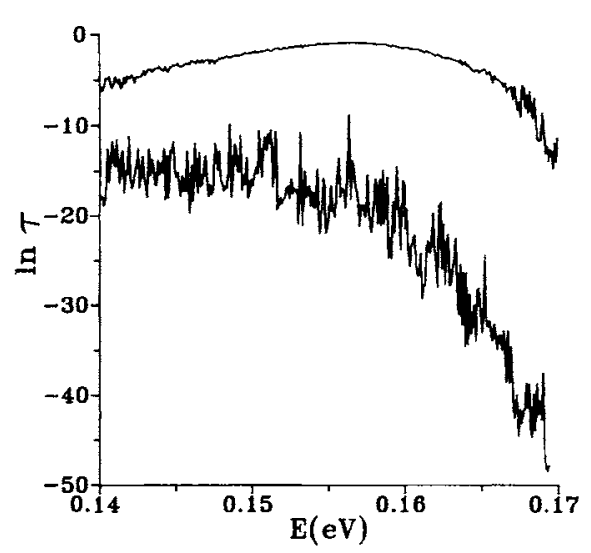

FIG. 1. Transmission coefficient around the resonant energy for a random QWSL (lower curve) and a DQWSL (upper curve). Shown are averages over 300 realizations, every SL consists of $N=500$ barriers of $b=200 \AA$ whereas the thicknesses of QW are $a=200 \AA$ and $a^{\prime}=160 \AA$. Note that the vertical axis is $\ln t$.

resonant energy $E_{r}$ within an allowed miniband of the periodic SL, that is, the resonant energy in the range of energies given by $\mathrm{Eq}$. (4).

\section{TRANSPORT THROUGH A DQWSL}

We now turn to the problem we are interested in, namely, SL's with a finite concentration of DQW's, to verify whether the single DQW resonance is still preserved. To this end, we apply the previous results to a specific case, namely, $\mathrm{GaAs} / \mathrm{Ga}_{0.65} \mathrm{Al}_{0.35} \mathrm{As}$. In this case $\Delta E_{c}=0.25 \mathrm{eV}$ and $m^{*}=0.067 m, m$ being the electron mass. In our computations we have taken $a=b=200 \AA$ and $a^{\prime}=160 \AA$. With these parameters we find from Eq. (4) only one allowed miniband below the barrier, ranging from $0.1022 \mathrm{eV}$ up to $0.1755 \mathrm{eV}$. The resonant energy is $E_{r}=0.1565 \mathrm{eV}$ from Eq. (6) and thus it lies in this allowed miniband. The maximum number of barriers we have considered is $N=1000$ and the number of wells with thickness $a^{\prime}$ is $N / 5$, although we have checked that the main conclusions of the present work are independent of this ratio. We have generated random SL's with and without the constraint of pairing, but always with the same number of wells of thickness $a^{\prime}$. The physical magnitudes we are interested in were averaged for several realizations of the SL's. The ensembles comprised a number of realizations varying from 200 up to 400 to test the convergence of the computed mean values, and this convergence was always satisfactory.

The transmission coefficient around the resonant energy is shown in Fig. 1 for SL's with $N=500$ barriers. We stress that, in spite of the fact that the plot corresponds to an average over 300 realizations of the SL's, the transmission coefficient for typical realizations behaves in the same way, although noisier. Close to the resonant energy there is an interval of energies that shows also very good transmission properties, similar to those of the resonant energy. This strong peak is not observed when DQW's are absent. Such a peak implies the appearance of a deep minimum in the Landauer resistance close to $E_{r}$, as it becomes evident from the relationship between $\tau$ and $\rho$. For brevity we do not show 


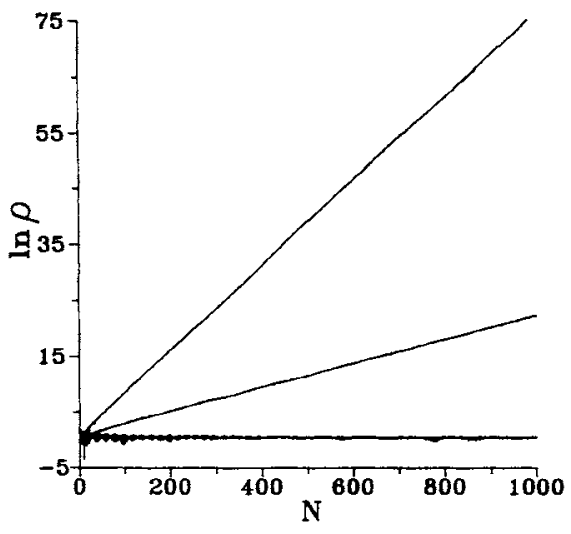

FIG. 2. Landauer resistance as a function of the number of barriers in $\mathrm{GaAs} / \mathrm{Ga}_{0.65} \mathrm{Al}_{0.35}$ As SL's with DQW for different energies: the resonant one $E_{r}=0.1565 \mathrm{eV}$ (lower curve), $0.9 E_{r}=0.1409 \mathrm{eV}$ (middle curve), and $0.8 E_{r}=0.1252 \mathrm{eV}$ (upper curve). Parameters are the same as in Fig. 1.

the corresponding figure, but it is worth mentioning that there are several orders of magnitude between the values of the resistance close to $E_{r}$ whether or not DQW's are present. In Fig. 2 we present the size dependence results for three different energy values (all of them lie in the allowed miniband) in random SL's with DQW. For those states with resonant energy $E_{r}$ the behavior is perfectly Ohmic, presenting only small fluctuations around the mean value. On the contrary, when we separate from the resonant energy we observe a non-Ohmic behavior of the resistance increasing exponentially with the system size: the more distant from the resonant energy, the larger the exponential growth of the resistance with the system size.

We now discuss the absence of localization by correlations in actual SL's, where imperfections during growth appear. We simulate excess or defect of monolayers during growth by allowing $\Delta x_{n}$ to fluctuate around the mean values $a+b$ or $a^{\prime}+b$. Therefore, $\Delta x_{n}=a\left(1+W \epsilon_{n}\right)+b$ or $\Delta x_{n}=a^{\prime}\left(1+W \epsilon_{n}\right)+b$, where $W$ is a positive parameter measuring the fluctuation and $\epsilon_{n}$ is chosen according to a uniform probability distribution $P\left(\epsilon_{n}\right)=1$ if $\left|\epsilon_{n}\right|<1 / 2$ and zero otherwise. It is important to stress that $\left\{\epsilon_{n}\right\}$ is a set of random uncorrelated variables, even when the lattice is constructed with the constraint that QW's with an average thickness $a^{\prime}$ always appear in pairs. Therefore, each QW presents a slightly different value of its thickness and, as a consequence, it should be expected that resonant coupling between electronic states of neighboring QW's decreases. Figure 3 shows the Lyapunov coefficient around the resonant energy $E_{r}$ for perfect and imperfect DQWSL's. A marked minimum is observed in all cases, even when fluctuations are relatively large, up to $10 \%$. We note that presently available molecular-beam epitaxy (MBE) techniques allow for growth control better than the previous fluctuations. In contrast, this minimum is absent in a random QWSL without imperfections. This means that a strong enhancement of the localization length close to the resonant energy is expected, even if there exists an uncorrelated disorder due to growth fluctuations. This is the reason why we claim that delocalization by

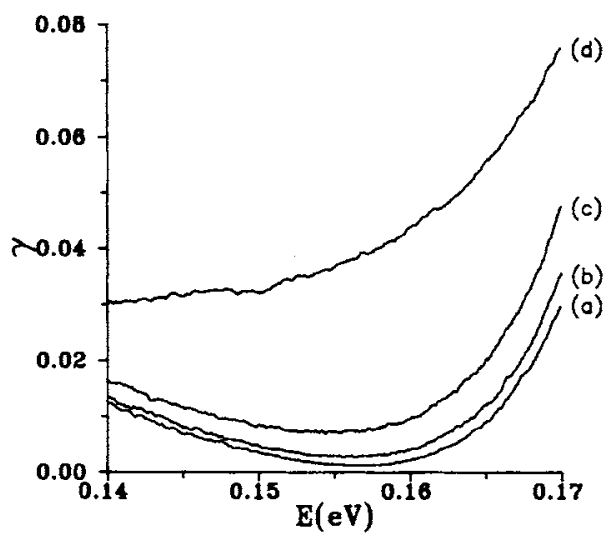

FIG. 3. Lyapunov coefficient around the resonant energy for a DQWSL with (a) $W=0$, (b) $W=0.05$, (c) $W=0.10$, and (d) random QWSL with $W=0$. Parameters are the same as in Fig. 1.

correlated disorder is a robust phenomenon and must be observed clearly in SL's.

\section{CONCLUSIONS}

We have studied electron transport in QW-based random SL's with and without DQW, showing that there exists a resonant energy for which a complete transparency through a single DWQ is achieved. This resonant energy depends only on structural parameters (layer thicknesses) in a given SL and, consequently, it is possible to place it within a miniband of the periodic SL. As a major point, we have found that these resonance effects also arise when a finite number of DQW are randomly placed in the SL, leading to very good transmission in a finite energy range around the resonant one. In a simple Kronig-Penney model with dimer impurities we have previously found ${ }^{9}$ that such minimum cause a dramatic enhancement of the dc conductivity at finite temperature whenever the Fermi level lies close to it. Our present results strongly support the idea that similar effects should be experimentally observable in actual SL's with correlated disorder. Moreover, states close to the resonant energy present Ohmic behavior, whereas when we deviate from this energy the resistance shows an exponential increase with the system size. Finally, such quasi-ballistic-electron states present a very large localization length, opposite to what occurs in random SL's without the constraint of pairing. We feel, however, that, the most important point we have demonstrated is that imperfections inadvertently introduced during growth, i.e., random variations of the ideal thicknesses of the QW's, have not very significant effects on the delocalization of electronic states by correlated disorder with the available MBE techniques. This is indeed an important result from a practical point of view since it means that deviations of a few monolayers from the ideal values of the well thicknesses cannot destroy the quantum coherence required to observe delocalization.

Experiments on such SL's would validate (or discard) all the recent claims that correlation induces the appearance of extended states in spite of disorder. A possible experimental setup is as follows. The random SL is inserted between two thick barriers doped with a high density of Si (typically 
$1 \times 10^{18} \mathrm{~cm}^{-3}$ ), so that the Fermi level is pinned at the dopant energy level (about $0.14 \mathrm{eV}$ in $\mathrm{Ga}_{0.65} \mathrm{Al}_{0.35} \mathrm{As}$ ). Different random SL's are prepared varying $a^{\prime}$ while keeping constant $a$ and $b$ (say $a=b=200 \AA$ as those SL's we have studied). By varying the value of $a^{\prime}$ the resonant energy is moved through the allowed miniband (for instance, $E_{r}$ ranges from $0.1187 \mathrm{eV}$ for $a^{\prime}=220 \AA$ up to $0.1643 \mathrm{eV}$ for $a^{\prime}=150 \AA$ ). Therefore, plots of the SL dc conductance at low temperature as a function of $a^{\prime}$ should exhibit a clear peak when the resonant energy matches the Fermi level. If this maximum is actually observed we will then be led to the conclusion than quasiballistic transport is taking place.

We hope that our results may encourage experimental effort in this direction for two reasons. First, and most importantly, to validate or not validate the existence of extended states in random systems with short-range correlations. Second, the feature of having quasiballistic transport only for certain energies may be the basis for designing new devices and applications.

\section{ACKNOWLEDGMENTS}

A.S. is thankful to Alan Bishop for warm hospitality at Los Alamos National Laboratory where this paper was written in part. Work at Madrid was supported by UCM through Project No. PR161/93-4811. Work at Leganés was supported by the DGICyT (Spain) through Project No. PB92-0248, and by the European Union Human Capital and Mobility Programme through Contract No. ERBCHRXCT930413.
${ }^{1}$ P. Phillips and H.-L. Wu, Science 252, 1805 (1991).

${ }^{2}$ A. Sánchez and F. Dominguez-Adame, J. Phys. A 27, 3725 (1994); A. Sánchez, E. Maciá, and F. Domínguez-Adame, Phys. Rev. B 49, 147 (1994); 49, 15 428(E) (1994).

${ }^{3}$ F. Domínguez-Adame, E. Maciá, and A. Sánchez, Phys. Rev. B 48, 6054 (1993).

${ }^{4}$ P. K. Datta and K. Kundu, J. Phys. Condens. Matter 6, 4465 (1994).

${ }^{5}$ S. N. Evangelou and A. Z. Wang, Phys. Rev. B 47, 13126 (1993).

${ }^{6}$ F. Domínguez-Adame, B. Méndez, A. Sánchez, and E. Maciá, Phys. Rev. B 49, 3839 (1994); F. Dominguez-Adame, E. Maciá, and A. Sánchez, ibid. 50, 6453 (1994).

${ }^{7}$ J. M. Ziman, Models of Disorder (Cambridge University Press, London, 1979).
${ }^{8}$ A. Chomette, B. Deveaud, A. Regreny, and G. Bastard, Phys. Rev. Lett. 57, 1464 (1986).

${ }^{9}$ E. Diez, A. Sánchez, and F. Domínguez-Adame, Phys. Rev. B 50, 14359 (1994).

${ }^{10}$ A. Sasaki, M. Kasu, T. Yamamoto, and S. Noda, Jpn. J. Appl. Phys. 28, L1249 (1989); M. Kasu, T. Yamamoto, S. Noda, and A. Sasaki, Appl. Phys. Lett. 59, 800 (1991); E. Tuncel and L. Pavesi, Philos. Mag. B 65, 213 (1992); X. Chen and S. Xiong, Phys. Rev. B 47, 7146 (1993); A. Wakahara, T. Hasegawa, K. Kuramoto, K. V. Vong, and A. Sasaki, Appl. Phys. Lett. 64, 1850 (1994).

${ }^{11}$ R. Landauer, IBM J. Res. Dev. 1, 223 (1957).

${ }^{12}$ P. D. Kirkman and J. B. Pendry, J. Phys. C 17, 4327 (1984). 